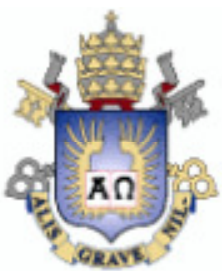

Nilmara Almeida Guimarães

\title{
Avaliação metrológica do tamanho de campo irradiado por aceleradores lineares
}

Dissertação de Mestrado

Dissertação apresentada como requisito parcial para obtenção do título de Mestre pelo Programa de Pós-Graduação em Metrologia da PUC-Rio (Área de concentração: Metrologia para Qualidade e Inovação).

Orientadora: Prof ${ }^{a}$. Dr ${ }^{a}$. Elisabeth Costa Monteiro Co-orientadora: Prof ${ }^{a}$. Dra . Cássia Ribeiro Ponciano 


\section{Pontificia U Uiversidade $\mathrm{C}_{\text {atólica }}$ \\ DO RIO DE JANEIRO}

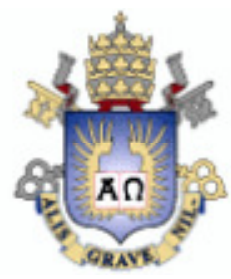

Nilmara Almeida Guimarães

\section{Avaliação metrológica do tamanho de campo irradiado por aceleradores lineares}

Dissertação apresentada como requisito parcial para obtenção do grau de Mestre pelo Programa de Pós-Graduação em Metrologia do Centro Técnico Científico da PUC-Rio. Aprovada pela Comissão Examinadora e homologada pela Coordenação Setorial de Pós-Graduação, formalizado pelas respectivas assinaturas:

Comissão Examinadora:

Prof ${ }^{a}$. Dr ${ }^{-}$. Elisabeth Costa Monteiro Orientadora

Programa de Pós-Graduação em Metrologia (PósMQI/PUC-Rio) Pontifícia Universidade Católica do Rio de Janeiro

Prof ${ }^{a}$. Drª . Cássia Ribeiro Ponciano Co-orientadora

Programa de Pós-Graduação em Metrologia (PósMQI/PUC-Rio) Pontifícia Universidade Católica do Rio de Janeiro

Prof. Dr. Carlos Roberto Hall Barbosa Programa de Pós-Graduação em Metrologia (PósMQI/PUC-Rio) Pontifícia Universidade Católica do Rio de Janeiro

Prof. Dr. Alfredo Viamonte Marin Programa de Qualidade em Radioterapia (PQRT do INCA/RJ) Instituto Nacional de Câncer do Rio de Janeiro

Prof. Dr. Vitor Luiz Bastos de Jesus Instituto Federal de Educação, Ciência e Tecnologia do Rio de Janeiro Campus Nilópolis (IFRJ-Nilópolis).

Coordenação Setorial de Pós-Graduação:

Prof. José Eugenio Leal Coordenador Setorial de Pós-Graduação do Centro Técnico Científico (PUC-Rio)

Rio de Janeiro, 01 de abril de 2011. 
Todos os direitos reservados. É proibida a reprodução total ou parcial do trabalho sem autorização da universidade, da autora e do orientador.

\title{
Nilmara Almeida Guimarães
}

Graduou-se em Tecnologia em Gestão da Produção e Metrologia no IFRJ (Instituto Federal de Educação, Ciência e Tecnologia) em 2009. Atuou como técnica em metrologia nos laboratórios de calibração acreditados do Centro de Pesquisas e Desenvolvimento Professor Leopoldo Américo Miguez de Mello - CENPES (2006 a 2009).

\section{Ficha Catalográfica}

\author{
Guimarães, Nilmara Almeida \\ Avaliação metrológica do tamanho de campo \\ irradiado por aceleradores lineares / Nilmara Almeida \\ Guimarães; orientadora: Elisabeth Costa Monteiro; co- \\ orientadora: Cássia Ribeiro Ponciano - 2011. \\ 143 f. : il. ; 29,7 cm \\ Dissertação (Mestrado em Metrologia) - Pontifícia \\ Universidade Católica do Rio de Janeiro, Rio de Janeiro, \\ 2011.
}

Inclui bibliografia

1. Metrologia - Teses. 2. Radioterapia. 3. Controle da Qualidade. 4. Acelerador Linear. 5. Tamanho de Campo. I. Monteiro, Elisabeth Costa. II. Ponciano, Cássia Ribeiro. III. Pontifícia Universidade Católica do Rio de Janeiro. Programa de Pós-Graduação em Metrologia para Qualidade e Inovação. IIII. Título.

CDD: 389.1 
Dedico este trabalho em primeiro lugar a Deus, meus pais Enilson e Maria, minha irmã Enimara, meu namorado Gustavo, meus cunhados Jefferson e Guilherme, meus sogros Marly e Ricardo, minhas grandes amigas Vanessa, Eduarda, Elazir, Jordana e Carmen. 


\section{Agradecimentos}

À minha orientadora Professora Elisabeth Costa Monteiro e à minha coorientadora e amiga Professora Cássia Ribeiro Ponciano pelo estímulo e parceria para a realização deste trabalho.

Ao CNPq, à PUC-Rio e ao PQRT do INCA, pelos auxílios concedidos, sem os quais este trabalho não poderia ter sido realizado.

Aos meus pais, pela educação, atenção e carinho de todas as horas.

Ao físico Roberto Salomon de Souza, pelas mais que importantes contribuições e apoio.

Aos físicos dos serviços de radioterapia que colocaram à disposição toda estrutura para que o principal objetivo deste trabalho fosse alcançado.

Aos meus colegas da PUC-Rio.

Aos professores que participaram da Comissão examinadora.

A todos os professores e funcionários do Departamento pelos ensinamentos e pela ajuda, em especial à secretária Márcia por todo o auxílio prestado e à assistente Paulinha pelo apoio.

A todos os amigos e familiares que de uma forma ou de outra me estimularam ou me ajudaram. 


\section{Resumo}

Guimarães, Nilmara Almeida; Monteiro, Elisabeth Costa; Ponciano, Cássia Ribeiro. Avaliação Metrológica do Tamanho de Campo Irradiado por Aceleradores Lineares. Rio de Janeiro, 2011. 143p. Dissertação de Mestrado - Programa de Pós-Graduação em Metrologia. Área de concentração: Metrologia para Qualidade e Inovação (PósMQI), Pontifícia Universidade Católica do Rio de Janeiro.

O objetivo desta dissertação é o estudo de procedimentos para avaliação da confiabilidade metrológica do tamanho de campo irradiado (TCI) por aceleradores lineares. No contexto das recentes alterações no panorama regulamentar dos serviços de radioterapia, com a implementação da $\mathrm{RDC}^{\circ} 20$, em 2006, pela Agência Nacional de Vigilância Sanitária (ANVISA), foi estabelecida a compulsoriedade do uso do densitômetro óptico na verificação do TCI, como parte do controle da qualidade. Questões associadas à implementação prática dos recentes requisitos para medição do tamanho de campo irradiado e as recentes indicações do potencial uso de filmes radiocrômicos para o controle da qualidade em radioterapia motivaram a realização do presente trabalho. Foram empregados três diferentes procedimentos para avaliação do tamanho de campo irradiado por aceleradores lineares utilizados em treze serviços de radioterapia localizados no Estado do Rio de Janeiro, utilizando filmes radiocrômicos EBT QD+, RTQA, densitômetro óptico DensiX tipo T52001 ou scanner de transmissão Microtek. Os resultados destacam a contribuição das fontes de incerteza de medição para cada procedimento realizado e indicam a necessidade de considerá-la na avaliação da conformidade utilizando o densitômetro óptico (DO). Devido às propriedades do filme radiocrômico RTQA, o mesmo não pode ser utilizado no procedimento de medição com DO. Em conclusão, o uso do procedimento de medição utilizando scanner mostrou-se mais adequado para avaliação das dimensões de campos de radiação não-homogêneos.

\section{Palavras-chave}

Radioterapia; Acelerador Linear; Controle da Qualidade; Tamanho de Campo Irradiado; Metrologia. 


\section{Abstract}

Guimarães, Nilmara Almeida; Monteiro, Elisabeth Costa (Advisor); Ponciano, Cássia Ribeiro (Co-advisor). Metrological evaluation of the radiation field size by linear accelerators. Rio de Janeiro, 2011. 143p. MSc. Dissertation - Programa de Pós-Graduação em Metrologia. Área de concentração: Metrologia para Qualidade e Inovação (PósMQI), Pontifícia Universidade Católica do Rio de Janeiro.

The objective of the present work is to study different procedures for metrological evaluation of the size of the radiation field emitted by linear accelerators. In the context of the recent requirements determined by the publication of the RDC $\mathrm{N}^{\circ} 20$, in 2006, by National Agency for Sanitary Vigilance (Anvisa), the compulsory use of the optical densitometer for the measurements of the field size during quality control tests was introduced. Practical implementation issues associated with these requirements and the recent studies indicating the potential use of radiochromic films for measurements of the field size motivated the present work. Three different procedures were employed in order to evaluate the radiation field size emitted by linear accelerators used in thirteen radiotherapy services located in Rio de Janeiro, using radiochromic films EBT QD+ and RTQA, optical densitometer DensiX type T52001 or scanner Microtek. The results indicate the contribution of the measurement uncertainty associated to each procedure performed, highlighting the importance of its consideration during tests for conformity assessment with the recently required optical densitometer. Nevertheless, the RTQA radiochromic film properties precluded its use in the procedure using optical densitometer. In conclusion, among the evaluated procedures for radiation field evaluation, the use of the scanner was the most appropriate, especially if dimensional non-homogeneities are present.

\section{Keywords}

Radiotherapy; Linear Accelerator; Quality Control; Radiation Field Size; Metrology. 


\section{Sumário}

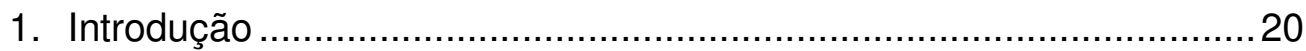

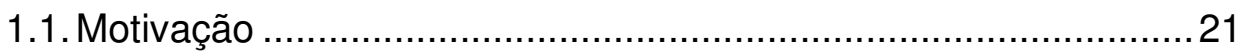

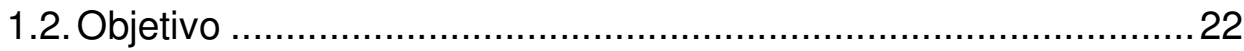

1.3. Metodologia ............................................................... 23

1.4. Estrutura da dissertação ................................................. 24

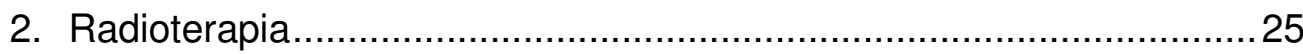

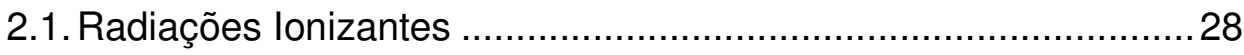

2.1.1.Efeitos Biológicos das Radiações lonizantes ..................... 30

2.2. Conceitos de Radioterapia .................................................... 31

2.3. Aceleradores de Partículas.................................................. 33

2.3.1.Aceleradores lineares utilizados em Radioterapia ................35

2.3.2.Sistema de Segurança do Acelerador Linear ..................... 42

3. Confiabilidade Metrológica em Radioterapia.................................... 44

3.1. Contexto Internacional ....................................................... 46

3.1.1.Bureau Internacional de Pesos e Medidas (BIPM) .............. 46

3.1.2. Organização Internacional de Metrologia Legal (OIML) .......51

3.1.3.Agência Internacional de Energia Atômica (AIEA)...............52

3.1.4.Comissão Eletrotécnica Internacional (IEC) ....................... 53

3.1.5.Organização Internacional para Padronização (ISO) ...........54

3.2. Contexto Nacional ............................................................... 54

3.2.1. Instituto Nacional de Metrologia, Normalização e Qualidade Industrial (INMETRO) ............................................... 54

3.2.2.Agência Nacional de Vigilância Sanitária (ANVISA) ............57

3.2.3.Comissão Nacional de Energia Nuclear (CNEN) .................58

3.2.4.Associação Brasileira de Normas Técnicas (ABNT) .............61

3.3. Controle da Qualidade em Radioterapia..................................61

3.3.1.Programa de Qualidade em Radioterapia - PQRT ..............63

3.3.1.1.Protocolo TECDOC-1151 ......................................... 64 


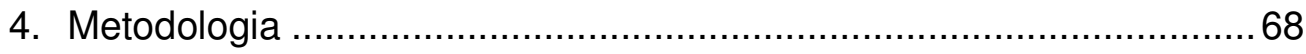

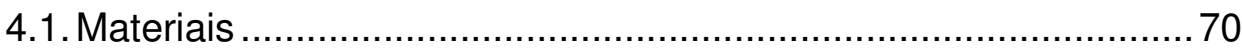

4.1.1.Filmes Radiocrômicos …………………………...................71

4.1.2.Densitômetro Óptico ......................................................... 73

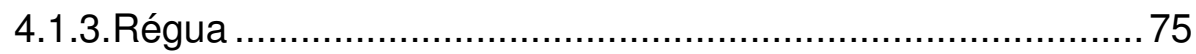

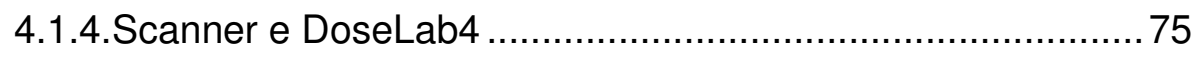

4.2. Avaliação do Tamanho de Campo Irradiado ................................ 78

4.2.1. Estimativa da incerteza de medição do tamanho de campo irradiado .85

4.2.1.1.Confiabilidade Metrológica do Tamanho de Campo Irradiado 94

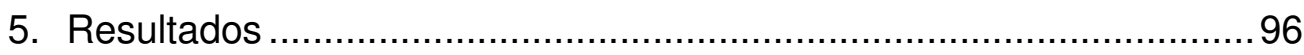

5.1. Erros no Tamanho de Campo Irradiado ................................................97

5.2. Incertezas de medição dos Tamanhos de Campos Irradiados estimadas para os procedimentos A, B e C ………………..........99

5.3. Confiabilidade Metrológica do Tamanho de Campo Irradiado.... 106

5.4. Contribuição da Incerteza de Medição na Avaliação da Conformidade 112

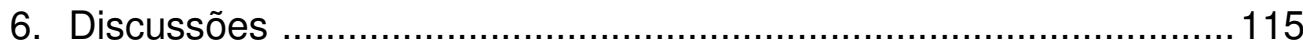

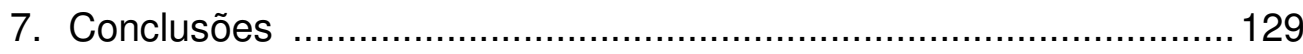

8. Referências Bibliográficas............................................................ 131

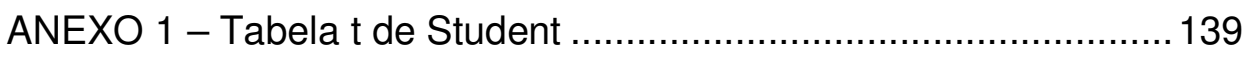

ANEXO 2 - Certificado de Calibração do Densitômetro ..................... 140

ANEXO 3 - Certificado de Calibração da Régua ............................... 141

APÊNDICE 1 - Formulário da Pesquisa de Campo.......................... 143 


\section{Siglas e Abreviaturas}

${ }^{60} \mathrm{Co}$ - Cobalto 60

a.C. - antes de Cristo

AAPM - Associação Americana de Físicos em Medicina

ABIFCC - Associação Brasileira das Instituições Filantrópicas de Combate ao Câncer

ABNT - Associação Brasileira de Normas Técnicas

ADN - Ácido Desoxirribonucléico

AIEA - Agência Internacional de Energia Atômica

AMN - Associação Mercosul de Normalização

Anvisa - Agência Nacional de Vigilância Sanitária

ARCAL - Acordo Regional de Cooperação para a promoção da ciência e tecnologia nucleares na América Latina e no Caribe

BIPM - Bureau Internacional de Pesos e Medidas

CBM - Comitê Brasileiro de Metrologia

CBTC - Comitê de Coordenação sobre Barreiras Técnicas ao Comércio

CCAB - Comitê Codex Alimentarius do Brasil

CGPM - Conferência Geral de Pesos e Medidas

CIPM - Comitê Internacional de Pesos e Medidas

CNA - Comissão Nacional de Acreditação

CNEN - Comissão Nacional de Energia Nuclear

CNES/MS - Cadastro Nacional de Estabelecimentos de Saúde do Ministério da Saúde

$\mathrm{CNI}$ - Confederação Nacional da Indústria

CNN - Comitê Nacional de Normalização

CONACRE - Antigo Comitê de Credenciamento de laboratórios e Organismos de Inspeção 
CONMETRO - Conselho Nacional de Metrologia, Normalização e Qualidade Industrial

COPANT - Comissão Panamericana de Normas Técnicas

CR - Comptes Rendus

DNA - Deoxyribonucleic Acid

DOU - Diário Oficial da União

EPID - Dispositivos eletrônicos de aquisição de imagem

IAEA - International Atomic Energy Agency

ICRU - Comissão Internacional de Unidades e Medidas da Radiação

IDEC - Instituto de Defesa do Consumidor

IEC - Comissão Electrotécnica Internacional

IFRJ-Nilópolis - Instituto Federal de Educação, Ciência e Tecnologia do Rio de Janeiro Campus Nilópolis

IM - Incerteza de Medição

INCA - Instituto Nacional de Câncer

INM - Instituto Nacional de Metrologia

INMETRO - Instituto Nacional de Metrologia, Normalização e Qualidade Industrial

IPEM - Instituto de Pesos e Medidas

IRD - Instituto de Radioproteção e Dosimetria

ISO - Organização Internacional para Padronização

ISO GUM - Guia para Expressão da Incerteza de Medição

ISP - International Specialty Products

Kp - Fator de abrangência

LIA - Limite Inferior de Aceitação

LIE - Limite Inferior de Especificação

LINAC - Linear Acceleratorl Acelerador Linear

LNMRI - Laboratório Nacional de Metrologia das Radiações Ionizantes 
LSA - Limite Superior de Aceitação

LSE - Limite Superir de Especificação

MCT - Ministério de Ciência e Tecnologia

MDIC - Ministério do Desenvolvimento, Indústria e Comércio Exterior

MLC - Colimador Multifolhas

MRA - Arranjo de Reconhecimento Mútuo

NBR - Norma Brasileira Registrada

OCC - Organismos de Certificação Acreditados

OIA - Organismos de Inspeção Acreditados

OIML - Organização Internacional de Metrologia Legal

OMS - Organização Mundial da Saúde

ONU - Organização das Nações Unidas

OPP - Organismo Provedor de Ensaio de Proficiência Acreditado

OTC - Organismos de Treinamento Credenciados

PDP - Percentual de Dose em Profundidade

PQRT - Programa da Qualidade em Radioterapia

PUC-Rio - Pontifícia Universidade Católica do Rio de Janeiro

RBC - Rede Brasileira de Calibração

RBLE - Rede Brasileira de Laboratórios de Ensaio

RDC - Resolução da Diretoria Colegiada

RGB - Red-Green-Blue

RM - Resultado de Medição

RMO - Organizações Regionais de Metrologia

SI - Sistema Internacional de Unidades

SINMETRO - Sistema Nacional de Metrologia, Normalização e Qualidade Industrial

SIR - Sistema Internacional de Referência 
DFS - Distância fonte-superfície

SUS - Sistema Único de Saúde

$\mathrm{TCl}$ - Tamanho de Campo Irradiado

TG - Task Group

TLC - Taxa de licenciamento, controle e fiscalização de materiais nucleares e radioativos e suas instalações

TNP - Tratado de Não-Proliferação de Armas Nucleares

TPR - Relação Tecido-Fantoma

TR - Informativo Técnico

TRS - Technical Reports Series

UM - Unidade Monitora

UV - Ultravioleta

$V_{\text {eff }}-$ Graus de Liberdade Efetiva

VIM - Vocabulário Internacional de Metrologia: Conceitos Fundamentais e Gerais e Termos Associados 


\section{Lista de figuras}

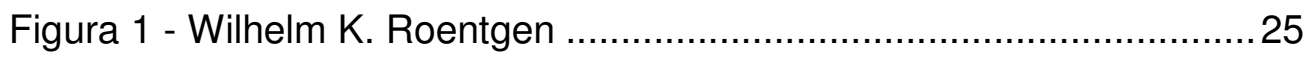

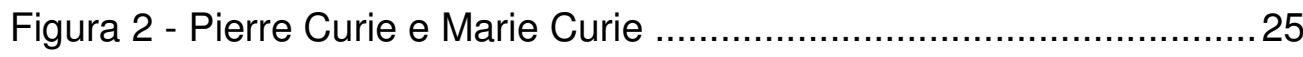

Figura 3 - Datas importantes na história da radioterapia......................... 28

Figura 4 - Classificação das radiações em função da interação com a

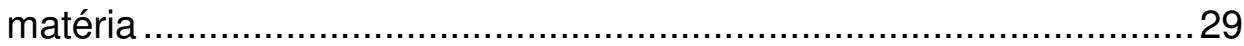

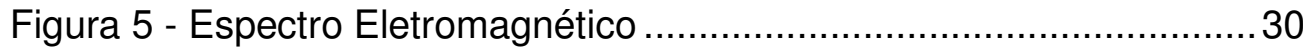

Figura 6 - llustração de um acelerador linear para radioterapia ...............35

Figura 7 - Histograma de Serviços de Radioterapia com Aceleradores

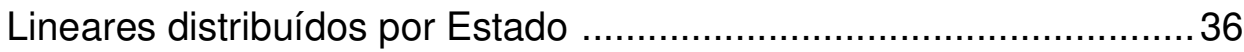

Figura 8 - Diagrama do acelerador linear .............................................. 36

Figura 9 - Vista em corte de uma guia de onda do acelerador linear de 6 MV

Figura 10 - Esquemas do cabeçote de um acelerador para produção de

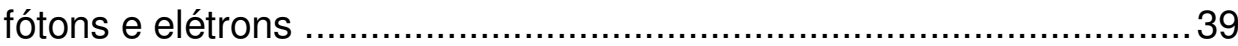

Figura 11 - Detalhes dos componentes do cabeçote de um acelerador linear

Figura 12 - Simulação de tamanhos de campo de radiação com colimador secundário formado por quatro blocos

Figura 13 - Colimador de múltiplas folhas no lado esquerdo e campo irregular formado pelas lâminas no lado direito

Figura 14 - Perfil do feixe utilizado para definição do tamanho de campo a $50 \%$ da dose irradiada 41

Figura 15 - Pirâmide de rastreabilidade ……………........................... 45

Figura 16 - Estrutura Metrológica do Brasil ...........................................56

Figura 17 - Camadas do filme Gafchromic RTQA ………......................72

Figura 18 - Camadas do filme Gafchromic EBT QD+ ............................ 72

Figura 19 - Densitômetro Óptico PTW-DensiX tipo T52001 ……..............74

Figura 20 - Sistema para aquisição da imagem do filme radiocrômico composto por um scanner Microtek e um PC ………….................... 76

Figura 21 - Tela do software ScanMaker: Seleção da imagem no filme radiocrômico para digitalização do campo irradiado ...........................76 
Figura 22 - Conversão do campo irradiado em escala de cinza para análise da dose e do tamanho de campo 77

Figura 23 - Posição de leitura do comprimento dos eixos $\mathrm{X}$ e $\mathrm{Y}$ do campo irradiado com uso da régua calibrada 80

Figura 24 - Método para medição do comprimento dos eixos $X$ e $Y$ com o uso do DoseLab 4 após definição dos pontos $(x, y)$ nas bordas do campo irradiado identificado pelas linhas tracejadas em cor vermelha

Figura 25 - Medição do comprimento do eixo Y central com o uso do DoseLab4 83

Figura 26 - Medição do comprimento do eixo X central com o uso do DoseLab4 84

Figura 27 - Diagrama de causa e efeito aplicado à incerteza de medição do tamanho de campo irradiado. 86

Figura 28 - Semelhança de triângulos para calcular a contribuição de incerteza da medição devido à diferença no valor da DFS

Figura 29 - Apresentação dos mensurandos no campo irradiado avaliados na repetitividade do procedimento de avaliação do tamanho de campo irradiado por aceleradores lineares

92

Figura 30 - Zona de Conformidade (ZC) estabelecido pelo TECDOC-1151 e utilizado na avaliação metrológica do tamanho de campo irradiado de $10 \mathrm{~cm} \times 10 \mathrm{~cm}$. 94

Figura 31 - Simulação do intervalo de aceitação considerado no presente trabalho, caso a estimativa da incerteza de medição do $\mathrm{TCl}$ seja de \pm $0,1 \mathrm{~cm}$ 95

Figura 32 - Valores médios do comprimento do eixo X e correspondente incerteza da repetitividade do método 100

Figura 33 - Valores médios do comprimento do eixo Y e correspondente incerteza da repetitividade do método 100

Figura 34 - Valores mínimos e máximos das estimativas de incerteza de medição obtidas na realização dos procedimentos $\mathrm{A}, \mathrm{B}$ e $\mathrm{C}$ utilizados para medição do comprimento do Eixo $X$ do campo irradiado pelos aceleradores lineares avaliados. 
Figura 35 - Valores mínimos e máximos das estimativas de incerteza de medição obtidas na realização dos procedimentos $A$, B e C utilizados para medição do comprimento do Eixo $Y$ do campo irradiado pelos aceleradores lineares avaliados

Figura 36 - Valores mínimos e máximos das estimativas de incerteza de medição obtidas na realização dos procedimentos $A, B$ e $C$ utilizados para medição do comprimento do Eixo $\mathrm{X}$ e $\mathrm{Y}$ do campo irradiado pelos aceleradores lineares avaliados. 105

Figura 37 - Faixa do resultado de medição do eixo $X$ do tamanho de campo irradiado, para cada acelerador avaliado segundo o Procedimento $\mathrm{A}_{\mathrm{EBT}} \mathrm{QD}+$

Figura 38 - Faixa do resultado de medição do eixo $Y$ do tamanho de campo irradiado, para cada acelerador avaliado segundo o Procedimento A $\mathrm{EBT}_{\mathrm{QD}+}$

Figura 39 - Faixa do resultado de medição do eixo $X$ do tamanho de campo irradiado, para cada acelerador avaliado segundo o Procedimento $A_{\text {RTQA }}$

Figura 40 - Faixa do resultado de medição do eixo $Y$ do tamanho de campo irradiado, para cada acelerador avaliado segundo o Procedimento $A_{\text {RTQA }}$ 108

Figura 41 - Faixa do resultado de medição do eixo $X$ do tamanho de campo irradiado, para cada acelerador avaliado segundo o Procedimento $\mathrm{B}_{\mathrm{EBT}} \mathrm{QD}+$

Figura 42 - Faixa do resultado de medição do eixo $Y$ do tamanho de campo irradiado, para cada acelerador avaliado segundo o Procedimento $\mathrm{B}_{\mathrm{EBT} \mathrm{QD}+}$

Figura 43 - Faixa do resultado de medição do eixo $X$ do tamanho de campo irradiado, para cada acelerador avaliado segundo o Procedimento $\mathrm{C}_{\mathrm{RTQA}}$

Figura 44 - Faixa do resultado de medição do eixo $Y$ do tamanho de campo irradiado, para cada acelerador avaliado segundo o Procedimento $\mathrm{C}_{\mathrm{RTQA}}$.

Figura 45 - Faixa do resultado de medição do eixo $X$ do tamanho de campo irradiado, para cada acelerador avaliado segundo o Procedimento $\mathrm{C}_{\mathrm{EBT}} \mathrm{QD}+$

Figura 46 - Faixa do resultado de medição do eixo $Y$ do tamanho de campo irradiado, para cada acelerador avaliado segundo o Procedimento $\mathrm{C}_{\mathrm{EBT}} \mathrm{QD}+$

Figura 47 - Faixa do resultado de medição do eixo $X$ do tamanho de campo irradiado, para o acelerador 1 avaliado por todos os procedimentos realizados

Figura 48 - Faixa do resultado de medição do eixo $X$ do tamanho de 
campo irradiado, para o acelerador 3 avaliado por todos os procedimentos realizados

Figura 49 - Faixa do resultado de medição do eixo $X$ do tamanho de campo irradiado, para o acelerador 7 avaliado por todos os procedimentos realizados

Figura 50 - Faixa do resultado de medição do eixo $X$ do tamanho de campo irradiado, para o acelerador 8 avaliado por todos os procedimentos realizados

Figura 51 - Faixa do resultado de medição do eixo $X$ do tamanho de campo irradiado, para o acelerador 9 avaliado por todos os procedimentos realizados

Figura 52 - Faixa do resultado de medição do eixo $X$ do tamanho de campo irradiado, para o acelerador 11 avaliado por todos os procedimentos realizados

Figura 53 - Faixa do resultado de medição do eixo $X$ do tamanho de campo irradiado, para o acelerador 12 avaliado por todos os procedimentos realizados

Figura 54 - Faixa do resultado de medição do eixo $X$ do tamanho de campo irradiado, para o acelerador 13 avaliado por todos os procedimentos realizados

Figura 55 - Faixa do resultado de medição do eixo $Y$ do tamanho de campo irradiado, para o acelerador 1 avaliado por todos os procedimentos realizados

Figura 56 - Faixa do resultado de medição do eixo $Y$ do tamanho de campo irradiado, para o acelerador 3 avaliado por todos os procedimentos realizados

Figura 57 - Faixa do resultado de medição do eixo $Y$ do tamanho de campo irradiado, para o acelerador 7 avaliado por todos os procedimentos realizados

Figura 58 - Faixa do resultado de medição do eixo $Y$ do tamanho de campo irradiado, para o acelerador 8 avaliado por todos os procedimentos realizados

Figura 59 - Faixa do resultado de medição do eixo $Y$ do tamanho de campo irradiado, para o acelerador 9 avaliado por todos os procedimentos realizados

Figura 60 - Faixa do resultado de medição do eixo $Y$ do tamanho de campo irradiado, para o acelerador 11 avaliado por todos os procedimentos realizados

Figura 61 - Faixa do resultado de medição do eixo $Y$ do tamanho de campo irradiado, para o acelerador 12 avaliado por todos os procedimentos realizados

Figura 62 - Faixa do resultado de medição do eixo $Y$ do tamanho de campo irradiado, para o acelerador 13 avaliado por todos os procedimentos realizados 


\section{Lista de quadros e tabelas}

Quadro 1 - Evolução dos aceleradores lineares para radioterapia.

Quadro 2 - Classificação dos Raios X em função das energias cinéticas 35

Quadro 3 - Cronograma de criação dos comitês consultivos 47

Quadro 4 -Unidades de base do SI 49

Quadro 5 -Exemplos de múltiplos e submúltiplos de unidade SI e de algumas outras unidades que podem ser usadas na Radioterapia .....50

Quadro 6 - Normas de radioproteção da CNEN 60

Quadro 7 -Testes de Garantia da Qualidade de Aceleradores Lineares .65

Quadro 8 - Serviços de radioterapia do Estado do Rio de Janeiro credenciados pela CNEN em dezembro de 2010

Tabela 1 - Dados técnicos referentes aos aceleradores lineares avaliados nos treze serviços de radioterapia 70

Tabela 2 - Caracterização dos procedimentos a partir de materiais utilizados para a realização da medição do tamanho de campo irradiado

Tabela 3 -Avaliação da repetitividade para cada procedimento realizado 92

Tabela 4 - Fontes de Incerteza consideradas no modelo para o balanço de incertezas dos Procedimentos A, B e C, utilizados para medição do tamanho de campo irradiado .93

Tabela 5 -Tipos de Procedimentos realizados para medição do tamanho de campo nas avaliações de cada acelerador linear 97

Tabela 6 - Erros médios do tamanho de campo irradiado, ao longo do eixo $X$, para cada acelerador linear avaliado 98

Tabela 7 - Erros médios do tamanho de campo irradiado, ao longo do eixo Y, para cada acelerador linear avaliado 99

Tabela 8 -Resultados obtidos ao longo do eixo $\mathrm{X}$ e $\mathrm{Y}$, por procedimento para cada acelerador linear avaliado.

Tabela 9 -Incertezas de medição do tamanho de campo irradiado, ao longo do eixo $\mathrm{X}$ e $\mathrm{Y}$, para cada acelerador linear avaliado 104 
Tabela 10 - Percentual do valor das incertezas de medição em relação ao erro máximo admissível de $2 \mathrm{~mm}$ apresentados para cada acelerador em função do eixo de campo irradiado e procedimento de medição

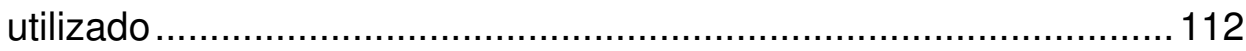

Tabela 11 -Resultado geral da pesquisa de campo ………………....113

Tabela 12 -Valores de incertezas de medição absolutas ....................... 116 